\title{
ANÁLISIS DE LA MARCHA Y EL EQUILIBRIO MEDIANTE EL USO DE SENSORES INERCIALES: ESTUDIO PROSPECTIVO, LONGITUDINAL, NO ALEATORIO
}

\author{
Gait and balance analysis through the use of inertial sensors. \\ Prospective, longitudinal, non-randomized study
}

\section{Frank Alberto Betances Reinoso a, Tania López Montes, Víctor Rodríguez Ontiveros y Carlos Chiesa Estomba}

\author{
Recibido: 20 de octubre, 2019 • Aprobado: 3 de enero, 2020
}

Cómo citar: Betances Reinoso FA, Lopez Montes T, Rodríguez Ontiveros VM, Chiesa Estomba C. Análisis de la marcha y el equilibrio mediante el uso de sensores inerciales: estudio prospectivo, longitudinal, no aleatorio. cysa [Internet]. 3 de marzo de 2020 [citado 4 de marzo de 2020];4(1):11-6. Disponible en: https://revistas.intec.edu.do/index.php/cisa/article/view/1671

Resumen

Antecedentes: la población mundial envejece de manera acelerada. Una tercera parte de los mayores de 65 años se cae por lo menos una vez al año, y esta prevalencia se incrementan a la mitad cuando se superan los 69 años.

Objetivo: establecer la sensibilidad del uso de sensores inerciales para determinar la marcha y las rotaciones del tronco en un grupo de individuos sanos.

Materiales: utilizamos tres sensores inerciales de nueve ejes, junto a un mismo número de microcontroladores, los cuales enviaron la información vía bluetooth al ordenador, el procesamiento de los datos fue realizado mediante un programa de análisis desarrollado en MATLAB. Para analizar la sensibilidad de los sensores utilizamos el protocolo de Akram et al., ${ }^{5}$ modificado, el cual consta de cinco actividades.

Resultados: en nuestro estudio participaron seis voluntarios, con una media de edad de 29 años, con un rango de edad de 25 a 33 ańos. Los sensores fueron capaces de

\footnotetext{
a Servicio de Otorrinolaringología, Hospital Universitario Donostia. San Sebastián, Gipuzkoa, España. orcid.org/0000-0002-6349-9537

Correo-e: drbetances@hotmail.com

* Conflicto de intereses: los autores declaran no tener conflicto de intereses.

b Servicio de Oftalmología, Royal Cornwall Hospital, Truro, Cornwall, Reino Unido. Correo-e: tania1_211m@hotmail.com
}

\begin{abstract}
Introduction: The world population is aging rapidly. A third of those over 65 years of age falls at least once a year, and this prevalence increases by half when they exceed 69 years.

Objective: To determine the sensitivity of the use of inertial sensors to determine the gait and rotations of the trunk in a group of healthy individuals.

Materials: We use 3 inertial sensors of 9-axis together with the same number of microcontrollers, that sent the information via bluetooth to the computer, the data processing was carried out through an analysis program developed in MATLAB. To analyze the sensitivity of the sensors we use the protocol of Akram et al, ${ }^{5}$ modified, which consists of 5 activities.

Results: In our study 6 volunteers participated, with an average age of 29 years, with an age range 25 to 33 years. The sensors were able to detect multiple variables, including the maximum and minimum elevation of the legs, number of steps, the degree of inclination of the trunk, rotation, speed and cadence.

\footnotetext{
${ }^{c}$ Escuela Universitaria Politécnica de Teruel. Teruel, Teruel, España. Correo-e: victor_rod_ont@hotmail.com

d Servicio de Otorrinolaringología, Hospital Universitario Donostia. San Sebastián, Gipuzkoa, España. Correo-e: drbetances@hotmail.com
} 
detectar múltiples variables, entre ellas la elevación máxima y mínima de las piernas, número de pasos, el grado de inclinación del tronco, giro, velocidad y cadencia.

Conclusión: los resultados hasta ahora obtenidos son prometedores y consideramos que el uso de múltiples sensores inerciales para valorar la marcha y el equilibrio podrían resultar en una nueva herramienta para el diagnóstico y seguimiento de personas con trastorno de la marcha y equilibrio.

Palabras clave: balance; marcha; caída; sensores inerciales; posturografía. vértigo.

\section{Introducción}

La población mundial, y sobre todo la de España, está envejeciendo de manera acelerada, para el año 2050 se espera que cerca del $30 \%$ de la población española sea mayor de 65 años ${ }^{1}$ Una tercera parte de los mayores de 65 ańos se cae por lo menos una vez al año, y esta prevalencia se incrementa al $50 \%$ cuando se superan los 69 años $^{2}$. Por lo que se espera que aumente la demanda de atención de trastornos relacionados con los problemas de la marcha y equilibrio.

La marcha es un movimiento cíclico que se compone de una secuencia de acontecimientos que tienen lugar entre el contacto inicial de un pie hasta el siguiente contacto. Perry ${ }^{3}$ definió unas fases, períodos y tareas que constituyen un marco teórico estructurado y sistemático que sirven para definir la marcha normal y patológica. El ciclo de la marcha se divide en dos fases (apoyo y oscilación), tres tareas (aceptación del peso, apoyo monopodal y avance de la extremidad) y ocho períodos (contacto inicial, respuesta a la carga, apoyo medio, apoyo terminal, preoscilación, oscilación inicial, oscilación media y oscilación terminal).

La valoración del control postural y el equilibrio es relevante para orientar las intervenciones terapéuticas. La detección de estos déficits es de utilidad
Conclusion: The results obtained are promising and we believe that the use of multiple inertial sensors to assess gait and balance could result in a new tool for the diagnosis and monitoring of people with gait and balance disorder.

Keywords: postural balance; vertigo, fall, inertia; posturography. dizziness.

como medida preventiva, en el riesgo de caídas, o como medida que ayude al pronóstico o intervención. Generalmente, las herramientas de valoración que se utilizan para el análisis del control postural y el equilibrio están basadas en test estandarizados (Timed Up and Go, Berg, Tinetti)

Los trastornos de la marcha y equilibrio no solo afectan negativamente la calidad de vida de los pacientes afectados, limitando su movilidad, sino también son responsables de las caídas y sus secuelas, disminuyendo su esperanza de vida.

El objetivo de nuestro estudio fue establecer la sensibilidad del uso de sensores inerciales para determinar la marcha y las rotaciones del tronco en un grupo de individuos sanos.

\section{Material y métodos}

El sistema de análisis de la marcha consta de tres sensores inerciales (MPU9250), cada sensor trae consigo un magnetómetro de tres ejes, un acelerómetro de tres ejes y un giroscopio de tres ejes, en total nueve ejes. Para la recolección de los datos cada sensor es controlado por un microcontrolador STM32F103C8T6, el cual envía la información vía bluetooth al ordenador. El microcontrolador procesa internamente los datos provenientes del sensor inercial para calcular la aceleración lineal y la orientación relativa al polo norte de la tierra. 
Los datos enviados por cada microcontrolador al ordenador son: aceleración lineal, aceleración gravitacional, velocidad angular y la orientación. A partir de estos datos se calculan algunos parámetros de la marcha como cadencia, número de pasos, postura, velocidad, giros, ángulos de elevación, zancada y duración del paso en segundos.

Para poder realizar el análisis de la marcha los sensores deben de ser colocados de la siguiente manera (figura 1):

- Uno será usado como referencia relativa local y estará ubicado en la parte baja de la espalda en la región sacra, con el objetivo de analizar el centro de gravedad.

- Los otros dos sensores deben de ir colocados en la parte posterior de la pierna (uno en cada pierna a la altura del tobillo).

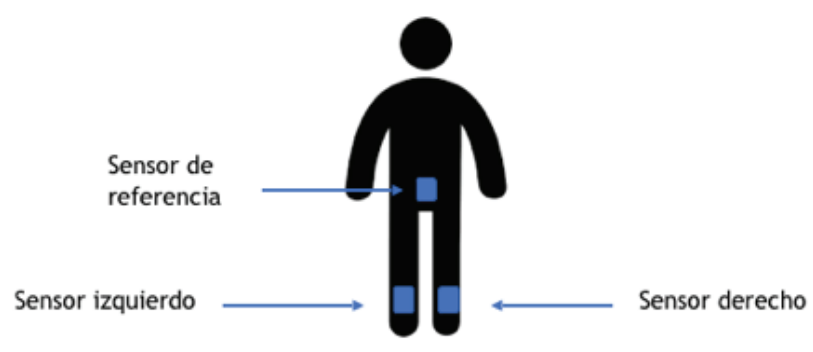

Figura 1. Distribución de los sensores inerciales

En nuestro caso, para analizar la sensibilidad de los sensores, utilizamos el protocolo de Akram et al. ${ }^{5}$ modificado, el cual consta de cinco actividades.

Utilizamos una cinta métrica para cuantificar la distancia a recorrer, en total diez metros, a su vez marcamos el lugar donde se realizaron los giros de 45 y 90 grados (figuras 2 y 3 ), utilizamos la cámara de un iPhone 8 para grabar cada actividad, y tener una prueba objetiva de los pasos y los giros. Cada participante realizó cinco actividades, dos giros de
45 grados, dos giros de 90 grados y, por último, un recorrido en línea recta.

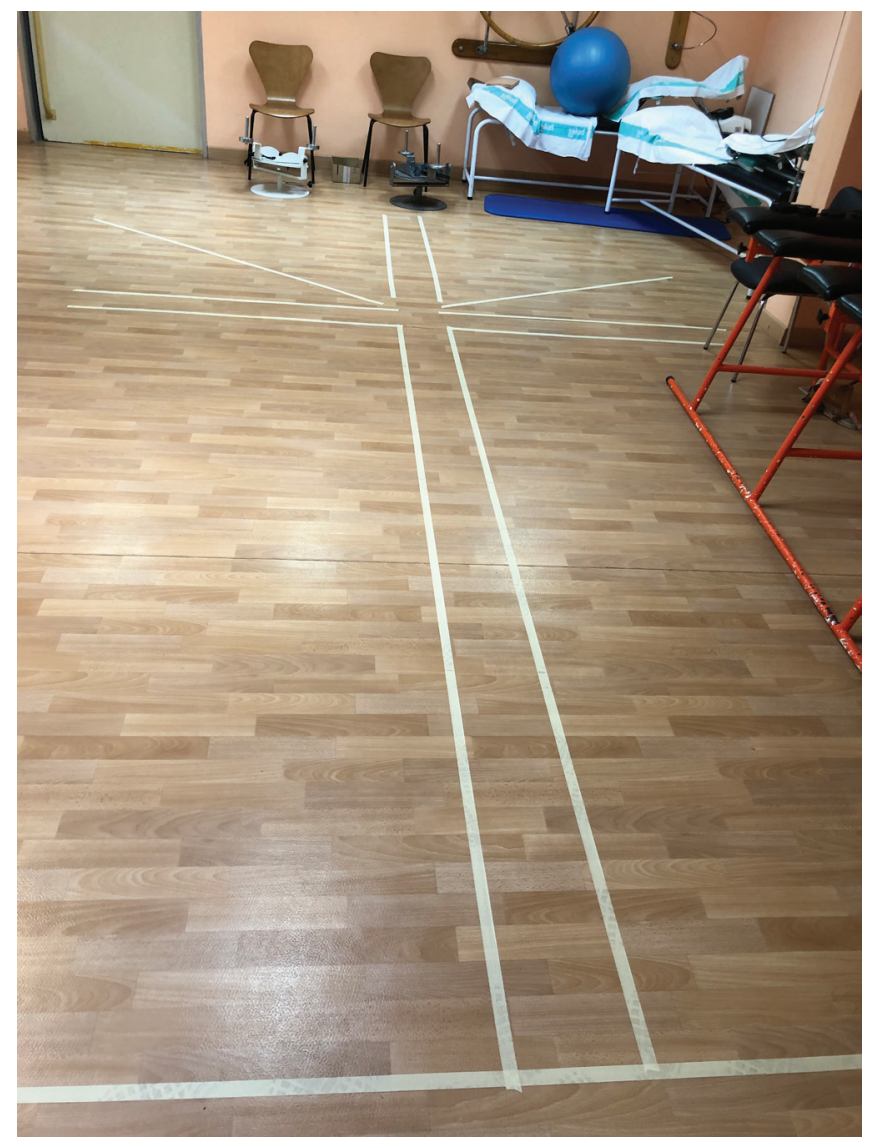

Figura 2. Distancia marcada con cinta métrica

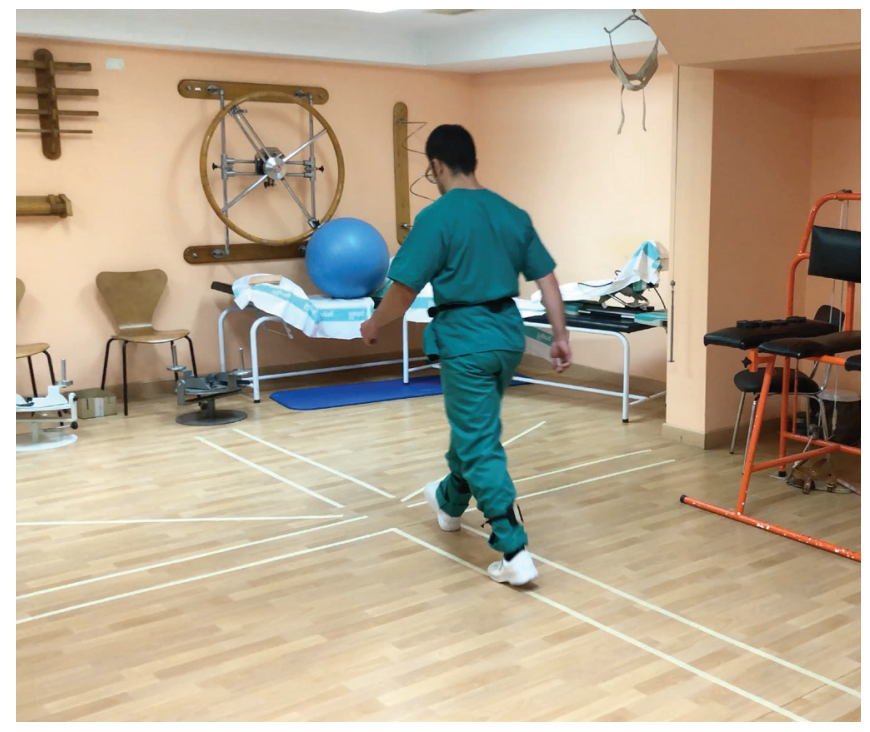

Figura 3. Voluntario completando actividades 


\section{Resultados}

En nuestro estudio participaron seis voluntarios, con una media de edad de 29 años y con un rango de edad entre 25 y 33 ańos, de los cuales cinco voluntarios fueron de sexo masculino y uno de sexo femenino. La altura promedio fue de $1,74 \mathrm{~m}$, con un rango de 1,60 a $1,85 \mathrm{~m}$. Los sensores fueron capaces de detectar múltiples variables, entre ellas la elevación máxima y mínima de las piernas, número de pasos, el grado de inclinación del tronco, giro, velocidad y cadencia. La media de pasos detectados en 10 metros de distancia fue de $13( \pm 2)$, con respecto al giro de 45 grados el dispositivo detectó un rango de giro de 44 a 47 grados, obteniendo un promedio de 45,1 grados. Al evaluar el giro de 90 grados, obtuvimos una media de 89,4 grado con un rango de 85,5 a 91 grados.

\section{Discusión}

Los problemas del equilibrio tienen una alta prevalencia, sobre todo en las personas mayores de 65 años, estos se ven obligados a generar y desarrollar estrategias durante la marcha para compensar el déficit de estabilidad, entre estas estrategias se encuentra acortar los pasos y desacelerar al caminar. Estas estrategias inicialmente consiguen mantener la estabilidad, pero terminan fracasando si no se realiza un adecuado diagnóstico e intervención sobre las causas que generan la falta de estabilidad, Akram et al. ${ }^{5}$ señalan que una de cada tres personas mayores de 65 años se cae al menos una vez al año; en Finlandia, las caídas y tropiezos fueron las causas más comunes y conducentes a la muerte, tanto en hombres como mujeres, durante el año $2010^{6}$.

El método más utilizado para valorar el equilibrio es la escala del equilibrio de $\mathrm{Berg}^{7}$ la cual consiste en una serie de 14 tareas donde se evalúa el desempeño de cada tarea mediante una escala de 0 a 4 , lo que hace que la puntuación máxima sea de 56 .
Las personas con una puntuación inferior a 49 muestran un mayor riesgo de caídas, mientras que las que tienen una puntuación superior a 49 tienen un bajo riesgo de caída. Sin embargo, la escala del equilibrio de Berg se basa en la evaluación subjetiva de, por ejemplo, un fisioterapeuta y proporciona información sobre el equilibrio en ese momento en particular, por lo que no es adecuada para la estimación continua del riesgo de caídas en situaciones de vida libre. Mediante el uso de sensores se elimina la subjetividad, al mismo tiempo permite evaluar de manera continua la estabilidad en ambiente reales, obteniendo datos adicionales que podrían conducir a desarrollar programas de rehabilitación ajustados a las características individuales de cada paciente.

Se han realizado múltiples estudios comparando la valoración de la escala de Berg, el test de Tinetti y el Timed up and go; primero, de manera objetiva con un sensor inercial, y luego, de manera subjetiva, encontrando diferencias mayores del $10 \%{ }^{8,9}$.

En el año 2014 Huang et al. ${ }^{10}$ desarrollaron un sistema basado el acelerómetro de un iPod con objetivo de cuantificar de manera objetiva el adecuado cumplimiento de los ejercicios de rehabilitación vestibular, y demostrando la fiabilidad del sistema, sobre todo a la hora de documentar la evolución de los pacientes. En nuestro caso, utilizamos múltiples sensores para incrementar la fiabilidad.

En el año 2015, Alberts et al., utilizaron el sensor triaxial de un iPad 2 para determinar la estabilidad postural, demostrando que el sensor fue capaz de realizar evaluaciones precisas de la misma ${ }^{11}$. Un factor a tener en cuenta es el tamańo del iPad, es un equipo con unas dimensiones considerables que dificulta su uso en pacientes con trastornos del equilibrio, nuestros dispositivos tienen medidas compactas de $3 \times 3 \times 1 \mathrm{~cm}$, sin riesgo de que estropee con una caída. 
Los estudios publicados previamente están limitados por el uso de un solo sensor, o enfocados exclusivamente al estudio de la marcha o el equilibrio, nunca ambos a la vez, por lo que decidimos probar si es posible valorar la marcha y el equilibrio a la vez, utilizando múltiples sensores inerciales triaxiales.

Girar mientras se camina es una maniobra común en la vida cotidiana. El giro es un componente desafiante de la locomoción que requiere la traslación y rotación del cuerpo hacia la nueva dirección de desplazamiento, a la vez que mantiene la estabilidad dinámica5. Nuestros sensores fueron capaces de detectar la elevación máxima y mínima de las piernas, número de pasos, el grado de inclinación del tronco, giro, velocidad y cadencia.

Nuestros resultados, aunque limitados por el tamaño muestral del estudio, nos inducen a pensar que los sensores inerciales podrían ser de utilidad durante la valoración de los trastornos del equilibrio, sobre todo en ambientes reales como pueden ser casos de presbivértigo o hipofunciones vestibulares en general.

Hace falta realizar más estudios para demostrar la utilidad real de estos dispositivos, a futuro esperamos ejecutar un estudio complementando el test de Unterberger y test Timed up and go en pacientes con inestabilidad.

\section{Conclusión}

Nuestro prototipo aún se encuentra en fase experimental de desarrollo, y se necesitan más pruebas que permitan evaluar la fiabilidad de los resultados. No obstante, creemos que los resultados hasta ahora obtenidos son prometedores y que el uso de múltiples sensores inerciales para valorar la marcha y el equilibrio podrían resultar en una nueva herramienta para el diagnóstico y seguimiento de personas con trastorno de la marcha y equilibrio.

\section{Bibliografía}

1. Fernadez, J.L.; Parapar, C; Ruiz, M. El envejecimiento de la población. Unidad de Análisis FGCSIC. Lychnos: Cuadernos de la Fundación General. 2010 Sep 2;(1): 6-11. Available from: http://www.fgcsic.es/lychnos/es_es/articulos/ envejecimiento_poblacion.

2. Lord S R, Sherrington C, Menz H. B. Falls in Older People: Risk Factors and Strategies for Prevention. Australasian Journal on Ageing. 2009 Feb;28: 47-8.

3. 3- Perry J. Gait Analysis: Normal and Pathological Function. J Sports Sci Med. 2010 Jun 1;9(2): 353.

4. Caña-Pino A, Apolo-Arenas MD, Moral-Blanco J, Álvaro-de Diego J, Fernández Gutiérrez C. Valoración del equilibrio postural en bipedestación-sedestación en sujetos sanos mediante acelerometría. Estudio piloto. Fisioterapia. 2015 Nov;37(6): 271-8.

5. Akram SB, Frank JS, Fraser J. Effect of walking velocity on segment coordination during pre-planned turns in healthy older adults. Gait \& Posture. 2010 junio;32(2): 211-4.

6. Statistics Finland. Accident Mortality in 1936 to 2010. [Online]. Available from: http://www. stat.fi/til/ksyyt/2010/ksyyt_2010_2011-12-16_ kat_005_en.html

7. Berg KO, Wood-Dauphinee SL, Williams JI, Maki B. Measuring balance in the elderly: validation of an instrument. Can J Public Health. 1992 Jul-Aug;83 Suppl 2: S7-11.

8. Greene BR, O'Donovan A, Romero-Ortuno R, Cogan L, Scanaill CN, Kenny RA. Quantitative falls risk assessment using the timed up and go test. IEEE Trans Biomed Eng. 2010 Dec;57(12): 2918-26. 
9. Doheny EP, Fan CW, Foran T, Greene BR, Cunningham C, Kenny RA. An instrumented sit-to-stand test used to examine differences between older fallers and non-fallers. Conf Proc IEEE Eng Med Biol Soc. 2011;2011: 3063-6.

10. Huang K, Sparto PJ, Kiesler S, Siewiorek DP, Smailagic A. iPod-based in-home system for monitoring gaze-stabilization exercise compliance of individuals with vestibular hypofunction. J Neuroeng Rehabil. 2014 Apr 21;11: 69.
11. Alberts JL, Hirsch JR, Koop MM, Schindler DD, Kana DE, Linder SM, et al. Using Accelerometer and Gyroscopic Measures to Quantify Postural Stability. Journal of Athletic Training. 2015 Jun;50(6): 578-88. 